\title{
Applied Computational Engineering in Magnetic Resonance Imaging: A Tumor Case Study
}

\author{
Carlo Ciulla ${ }^{1}$, Dijana Capeska Bogatinoska ${ }^{2}$ \\ University for Information Science \& Technology, "St. Paul the Apostle", Partizanska B.B., 6000 Ohrid, Macedonia \\ ${ }^{1}$ Email: carlo.ciulla@uist.edu.mk, cxc2728@njit.edu \\ ${ }^{2}$ Email: dijana.c.bogatinoska@uist.edu.mk
}

Filip A. Risteski ${ }^{3}$ and Dimitar Veljanovski ${ }^{4}$

Skopje City General Hospital, Pariska B.B., 1000 Skopje, Macedonia

${ }^{3,4}$ Email: \{risteskifilip, dveljanovski\} @ bolnica.org.mk

\begin{abstract}
This paper solves the biomedical engineering problem of the extraction of complementary and/or additional information related to the depths of the anatomical structures of the human brain tumor imaged with Magnetic Resonance Imaging (MRI). The combined calculation of the signal resilient to interpolation and the Intensity-Curvature Functional provides with the complementary and/or additional information. The steps to undertake for the calculation of the signal resilient to interpolation are: (i) fitting a polynomial function to the signal, (ii) the calculation of the classic-curvature of the signal, (iii) the calculation of the Intensity-Curvature term before interpolation of the signal, (iv) the calculation of the Intensity-Curvature term after interpolation of the signal, (v) the solution of the equation of the two aforementioned Intensity-Curvature terms of the signal provides with the signal resilient to interpolation. The Intensity-Curvature Functional is the result of the ratio between the two Intensity-Curvature terms before and after interpolation. Because of the fact that the signal resilient to interpolation and the Intensity-Curvature Functional are derived through the process of re-sampling the original signal, it is possible to obtain an immense number of images from the original MRI signal. This paper shows the combined use of the signal resilient to interpolation and the Intensity-Curvature Functional in diagnostic settings when evaluating a tumor imaged with MRI. Additionally, the Intensity-Curvature Functional can identify the tumor contour line.
\end{abstract}

Index Terms-Applied Computational Engineering, Classic-Curvature, Intensity-Curvature term, IntensityCurvature Functional, Polynomial Function, Re-sampling, Signal Resilient to Interpolation.

\section{INTRODUCTION}

\section{A. Literature and Background}

The research presented here addresses the problem of the calculation of the signal resilient to interpolation [1] and the Intensity-Curvature Functional $[1,2]$ when studying the original signal of the Magnetic Resonance Imaging (MRI) of a tumor in the human brain.

Advantages are brought to light through the combined use of the signal resilient to interpolation and the Intensity-Curvature Functional when processing the MRI images. More specifically, the signal resilient to interpolation is able to provide with complementary specular information of the MRI images and such property is novel in literature. On the other hand, the Intensity-Curvature Functional is able to provide with the advantage consisting of the identification of the tumor contour line, which is a signal processing task closely related to segmentation. Image segmentation has received an enormous attention in the literature and so various techniques have been reported. For instance, an approach based on multilevel thresholds [3] uses the fast convergence of Particle Swarm Optimization (PSO), together with the jumping property of simulated annealing.

The discipline treated in the present manuscript, intersects with both applied mathematics and biomedical image processing. In relationship to the aforementioned intersection it is due to report samples of literature related to the topic herein addressed. For example brain tumor classification has been performed with an artificial neural network in [4]. It has been reported an approach which uses a modified active control method in order to perform left ventricle segmentation in MRI [5]. Image segmentation applied to MRI has been performed using immune-kernels modeled with infinite Gaussian mixture models [6]. While, MRI tumor segmentation has been performed with several different techniques corroborated by preprocessing steps [7], fractal based methodologies have been used to detect and segment the tumor from MRI $[8,9]$. Also, human brain tumor boundary detection from MRI has been performed using a Hopfield neural network [10]. In this paper, the properties of the Intensity-Curvature Functional to detect and to segment the tumor contour line from MRI images has been observed when using the bivariate linear model function (see for instance Fig. 7).

Automatic tumor MRI segmentation has been developed on the basis of a spatial probabilistic atlas 
containing knowledge about brain structures and the result of the segmentation has been compared to manual and semi-automated segmentation procedures [11]. An application of automatic tumor segmentation, which combines knowledge based (KB) techniques with multispectral analysis has been reported in [12]. The multispectral analysis is used to target the suspected tumor in MRI images. Another approach to the semiautomatic tumor brain segmentation from MRI images uses a combination of: (i) intensity-based fuzzy classification of voxels into tumor, and (ii) the signal processing techniques called snake [13]. A different approach to tumor segmentation uses the hypothesis that segmentation can be performed while processing the healthy brain structures instead of the areas of the brain affected by the tumor [14]. The aforementioned study aimed to distinguish between deviations (affected regions of the brain) from normalcy (healthy tissues). Other approaches reported in literature show that tumor segmentation can be achieved: (i) from contrast enhanced MRI [15, 16], (ii) using a watershed algorithm [17], and (iii) using a fuzzy Markovian Method [18]. At the aim to perform MRI human brain segmentation, an extension to the traditional fuzzy c-means (FCM) clustering algorithm was developed in [19] with the optimization aid of an artificial neural network; and a novel hidden Markov random field based approach was also reported in [20].

\section{B. The Applied Computational Enginering}

An important aspect of the mathematical procedure employed to calculate both of the signal resilient to interpolation and the Intensity-Curvature Functional is resampling. Re-sampling is used in order to calculate the value of the signal, through the polynomial function, at the intra-node coordinate.

The signal resilient to interpolation and the IntensityCurvature Functional results from the following mathematical procedure applied to the original MRI.

The first step is that one of fitting a model polynomial function to the MRI signal. The first step is necessary in order to build the continuum from a discontinuous sequel of sampled signal intensity values.

The second step is that one of the calculation of the Classic-Curvature of the MRI signal and such calculation is performed through the computation of all of the second-order derivatives of the Hessian of the model polynomial function fitted to the signal data. The result of the second step is the Classic-Curvature of the MRI signal, calculated through the sum of all of the aforementioned second-order derivatives.

The third step is that one of the calculation of the Intensity-Curvature term before interpolation which is the math expression of the integral of the product between: (i) the value of the signal and (ii) the value of the ClassicCurvature. In the calculation of the Intensity-Curvature term before interpolation both of the signal and the Classic-Curvature are calculated (through the polynomial function) at the node of the sampling grid.

The fourth step consists of the calculation of the Intensity-Curvature term after interpolation. Such term is obtained through the integral of the product between: (i) the value of the signal and (ii) the Classic-Curvature. In the calculation of the Intensity-Curvature term after interpolation both of the signal and the Classic-Curvature are calculated at the re-sampling coordinate chosen in between the nodes of the sampling grid.

The Intensity-Curvature Functional is the result of the ratio between the: (i) the Intensity-Curvature term before interpolation, and (ii) the Intensity-Curvature term after interpolation.

The fifth and conclusive step consists in the equation of the Intensity-Curvature term before interpolation and the Intensity-Curvature term after interpolation. The solution of the aforementioned equation provides with the math expression of the signal resilient to interpolation. The works reported in $[1,2]$ provides the reader with the complete mathematical procedure to calculate both of the signal resilient to interpolation and the IntensityCurvature Functional.

A concept is due of clarification. The signal resilient to interpolation does not intend to convey the meaning that the new signal resulting from the original signal is unaffected by any interpolation procedures. The word resilient ought to be such to convey the meaning 'bounce back' or 'to go up again', and this signifies that the signal resilient to interpolation is obtained from the signal from which is calculated, which is the original signal. Resampling the original signal can be obtained through interpolation and is thus subject to detriments.

The overall procedure for the calculation of the signal resilient to interpolation and the Intensity-Curvature Functional relies on the re-sampled signal. The choice of the re-sampling coordinate is therefore relevant to the appearance of the signal resilient to interpolation and the Intensity-Curvature Functional.

One more important aspect of the signal resilient to interpolation and the Intensity-Curvature Functional is their domain of application. This study shows that the combined use of the signal resilient to interpolation and the Intensity-Curvature Functional yields the applied computational engineering solution of the problem of adding complementary and/or additional information to the MRI signal when studying a tumor case. Along the line of thought that relies on the hypothesis that in biomedical diagnostic settings it is possible, through mathematical engineering procedures applied to the Magnetic Resonance Signal, to obtain additional and/or complementary information, the study herein presented, show that the hypothesis is true when using the signal resilient to interpolation and the Intensity-Curvature Functional.

In the theory section of this paper, the fundamental equations for the calculation of the signal resilient to interpolation and the Intensity-Curvature Functional are presented. In the results section the signal resilient to interpolation and the Intensity-Curvature Functional of the human brain tumor imaged with MRI are presented. In the discussion section, the theoretical implications of the combined use of both of the signal resilient to interpolation and the Intensity-Curvature Functional of 
the MRI are outlined, and in the conclusion section benefits and limitations are stated.

\section{THEORY}

Let $\mathrm{f}(\mathbf{x})$ be a polynomial function in $\mathrm{n}$ dimensions, with $\mathrm{n}=1 \ldots \mathrm{k}$, where $\mathrm{k}$ is an integer positive number.

$$
\mathrm{f}(\mathbf{x})=\mathrm{f}(\mathbf{0})+\zeta(\mathbf{x})
$$

Let the Hessian of the polynomial function be defined as:

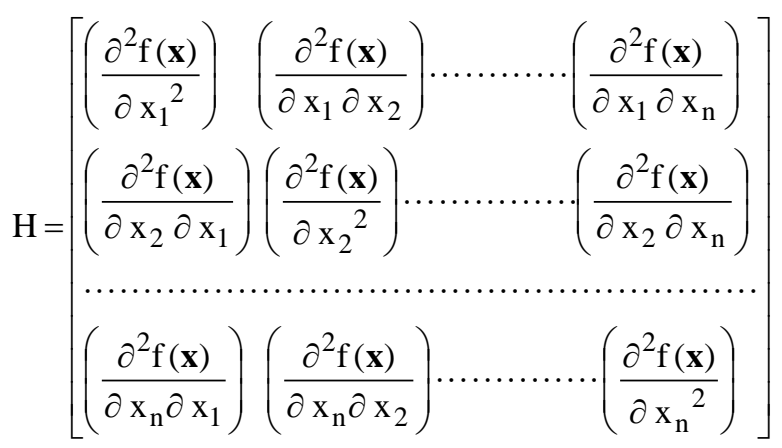

Let the Classic-Curvature of the polynomial function be defined as:

$$
\Omega(\mathbf{x})=\sum_{i, j=1}^{n}\left(\frac{\partial^{2} f(\mathbf{x})}{\partial x_{i} \partial x_{j}}\right)
$$

Let the Intensity-Curvature terms before interpolation (E0) and after interpolation (EIN) be defined through equations (4) and (5) respectively:

$$
\begin{aligned}
& \mathrm{E}_{0}=\mathrm{E}_{0}(\mathbf{x})=\int_{0}^{\mathrm{x}_{1}} \ldots \int_{0}^{\mathrm{x}_{\mathrm{n}}} \mathrm{f}(\mathbf{0}) \cdot \Omega(\mathbf{x})_{(\mathbf{0})} \mathrm{dx}_{1} \ldots \mathrm{dx}_{\mathrm{n}} \\
& \mathrm{E}_{\mathrm{IN}}=\mathrm{E}_{\mathrm{IN}}(\mathbf{x})=\int_{0}^{\mathrm{x}_{1}} \ldots \int_{0}^{\mathrm{x}_{\mathrm{n}}} \mathrm{f}(\mathbf{x}) \cdot \Omega(\mathbf{x})_{(\mathbf{x})} \mathrm{dx}_{1} \ldots \mathrm{dx}_{\mathrm{n}}
\end{aligned}
$$

Solving the equation $\mathrm{E} 0=\mathrm{EIN}$ in $\mathrm{f}(0)$ yields the signal resilient to interpolation:

$$
\mathrm{f}(\mathbf{0})=\mathrm{F}\left(\mathrm{E}_{0}(\mathbf{x})=\mathrm{E}_{\mathrm{IN}}(\mathbf{x})\right)=\mathrm{F}(\mathbf{x})
$$

The signal resilient to interpolation is a function of the variable $\mathrm{x}$, therefore changing in between the intra node coordinates. The signal resilient to interpolation can be calculated under the assumption that the model polynomial function fitted to the original signal benefits of the property of second-order differentiability in its interval of definition. The definition of the IntensityCurvature Functional is:

$$
\Delta \mathrm{E}(\mathbf{x})=\frac{\mathrm{E}_{0}(\mathbf{x})}{\mathrm{E}_{\mathrm{IN}}(\mathbf{x})}
$$

\section{RESULTS}

\section{A. The Signal Resilient to Interpolation}

This section shows the comparison of the original MRI with the signal resilient to interpolation obtained using a bivariate cubic Lagrange polynomial fitted to the image data and when re-sampling of $(\mathrm{x}, \mathrm{y})=(0.01 \mathrm{~mm}, 0.01 \mathrm{~mm})$

Figs. 1 through 4 illustrate the difference between the previously stated two sets of images when introducing the benefit provided through the signal resilient to interpolation. The bivariate cubic Lagrange polynomial is parametric in the value of the constant used in the convolution of the pixel intensity values and the value of the constant was set to -2.54 . Fig. 1 demonstrates that the signal resilient to interpolation can have a dual purpose. One purpose is to present the specular negative of the original MRI (see Fig.1b versus Fig. 1a, and Fig. 1d versus Fig. 1c). The other purpose is that one of highlighting the tumor features. The comparison of Fig. 1a and Fig. 1b, show the contour line of the tumor along with the tumor inner structures which are more visible in Fig. 1b. Specifically, the tumor inner structures are colored in bright and dark. The pressure that the tumor exercises on the ventricles is also visible. It is due to remark that the images in Fig. 1 have been brightnesscontrast adjusted so to reveal the details. And the observation of the images is in favor to the signal resilient to interpolation in a two folded manner.
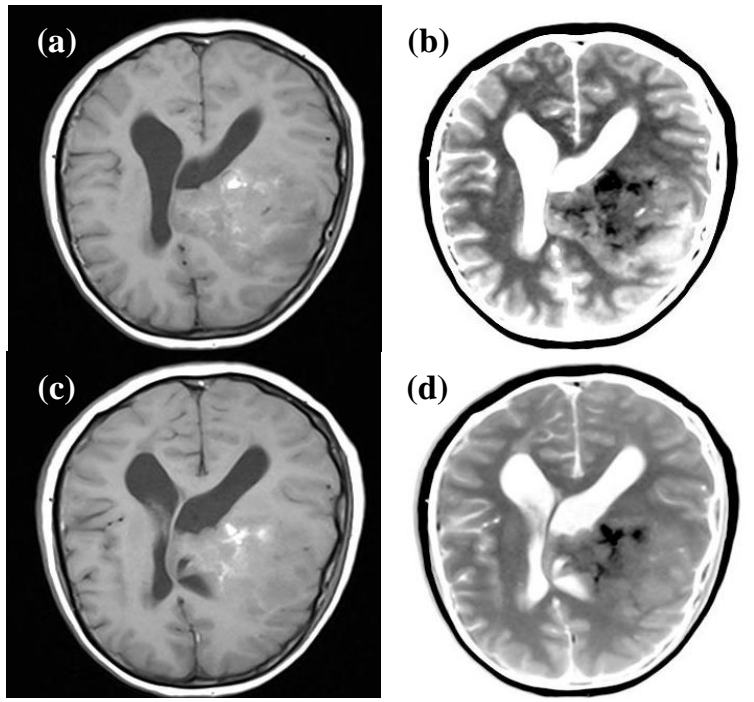

Fig 1: The tumor MRI is shown in (a) and in (c), the matrix size is $512 \times 512$ pixels with $0.49 \mathrm{~mm} \times 0.49 \mathrm{~mm}$ as pixel size. In (b) and in (d) is shown the signal resilient to interpolation of the images seen in (a) and in (c) respectively, with a visible highlight on the region of the tumor with the affected area characterized by the dark regions most likely showing fluids like blood and water. The images were cropped so to show the regions of interest.

One is that the distinction between gray and white matter of the brain is more visible in the signal resilient to interpolation images in (b) and in (d); and the other one is that the observation of (a) versus (b) and of (c) versus (d) demonstrates higher level of details in the signal resilient to interpolation images which would not be otherwise observable in the original MRI. The aforementioned 
observation is more evident when looking at the images in (a) and in (b) of Fig. 1.

Fig. 2 serves the purpose to illustrate that, with brightness-contrast enhancement set to a particular level, it is possible for the signal resilient to interpolation images (see Fig. 2b and Fig. 2d) to display in negative, the same level of details of the original MRI. The contour line of the tumor is well discernible in (a) and in (b) along with the details relevant to the fluids shown in bright and dark in the two images. It is interesting to note the slight difference between the image in Fig. 2a and the image in Fig. 2b, specifically the tumor structure seen in (a) is missing at the location indicated by the dark arrow in (b). In both of the images seen in (c) and in (d) the region of the tumor is well demarcated showing the affection of the

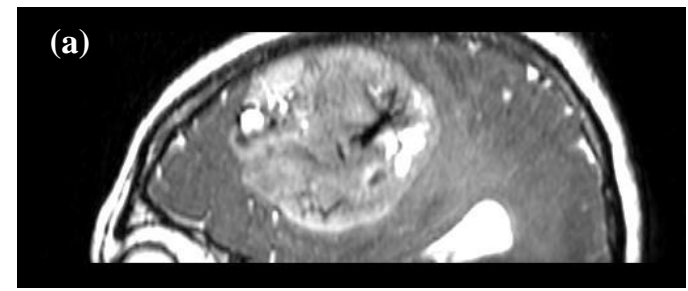

(b)
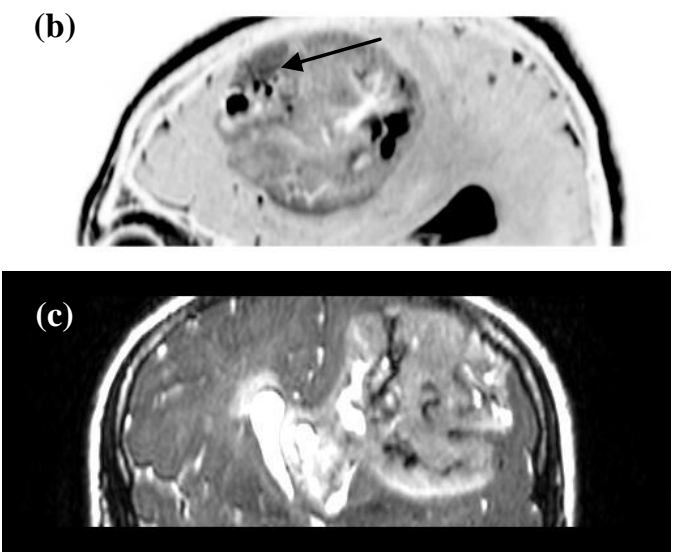

(d)

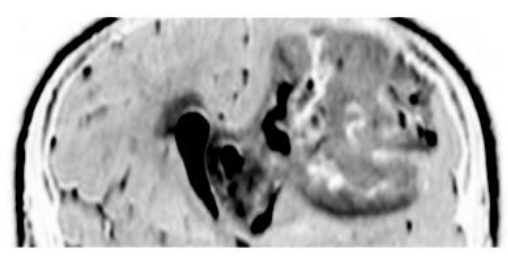

Fig 2: The tumor MRI is shown in (a) and in (c), whereas the signal resilient to interpolation is shown in (b) and in (d). The matrix size is $512 \times 512$ pixels with $0.39 \mathrm{~mm} \times 0.39 \mathrm{~mm}$ as pixel size. Likewise in Fig. 1 , data in (a) and in (c) are fitted with the bivariate cubic Lagrange model function and re-sampled with $(\mathrm{x}, \mathrm{y})=(0.01 \mathrm{~mm}, 0.01 \mathrm{~mm})$. The signal resilient to interpolation highlights on the region of the tumor with specific focus on the fluids which are shown in dark and in bright in the images. In this figure though the signal resilient to interpolation acts as a negative of the original MRI, and the level of details of the signal resilient to interpolation is not superior to the MRI of (a) and (c).

The images were cropped so to focus on the regions of interest.
Ventricles because of the presence and the extension of the tumor. In summary, Fig. 2 is a case in which is objective to report that the signal resilient to interpolation offers complementary information but not additional information in reference to the original MRI.

Fig. 3 shows a typical case of complementariness between the signal resilient to interpolation and the MRI. The brightness-contrast enhancement has been set to values making a comparison which is on the same basis of objectiveness. While looking at the MRI of Fig. 3a and Fig. $3 c$ the following observations can be made. The extension of the tumor, the pressure exercised by the tumor onto the ventricles, the fluids which are presumably water and/or blood, and the nuances of the region of the tumor. Within the context of complementariness, the signal resilient to interpolation shown in Fig. 3b and in Fig. 3d confirms the anomaly seen in MRI. Specifically, when looking at the regions inside the white circles in Fig. 3 it is possible to have confirmation of the extension of the tumor in reference to the tumor contour line (see bottom part of the image located inside the circles).

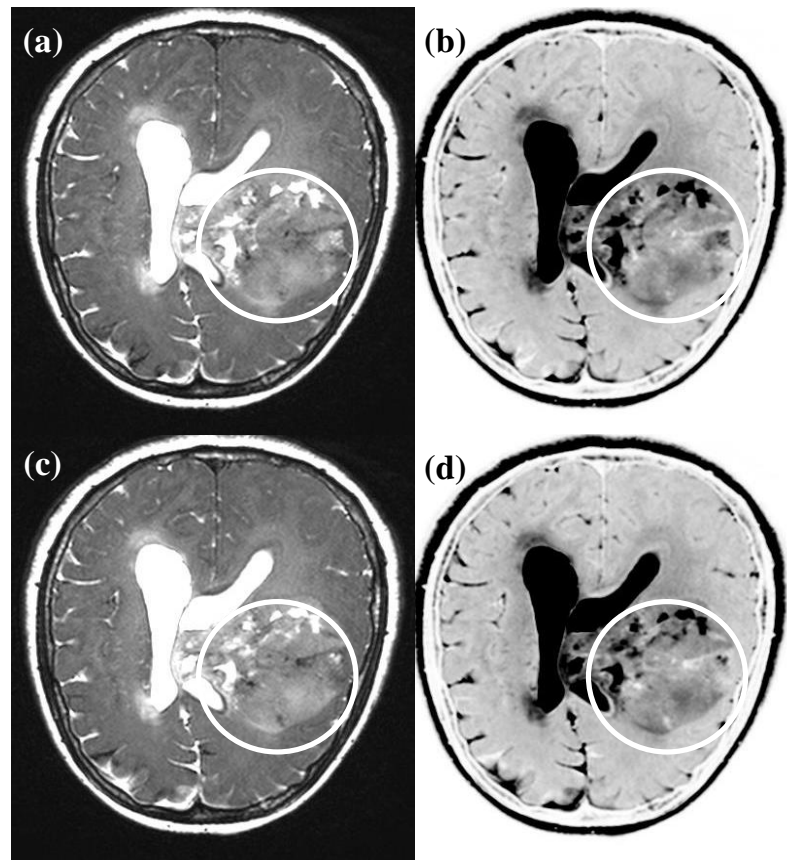

Fig 3: The MRI with the tumor is shown in (a) and in (c), the signal resilient to interpolation is shown in (b) and in (d). The complementary information provided by the signal resilient to interpolation focuses on the bright and dark regions of the tumor seen inside the circles, most likely fluids like blood and/or water. The level of details seen in (b) and in (d) is similar to the original MRI. The matrix size is $512 \times 512$ pixels with $0.39 \mathrm{~mm} \times 0.39 \mathrm{~mm}$ as pixel size. The images were cropped so to focus on the regions of interest. The model polynomial function fitted to the images shown in (a) and in (c) is the bivariate cubic Lagrange and the intra-pixel re-sampling coordinate is $(\mathrm{x}, \mathrm{y})=(0.01 \mathrm{~mm}, 0.01 \mathrm{~mm})$. 

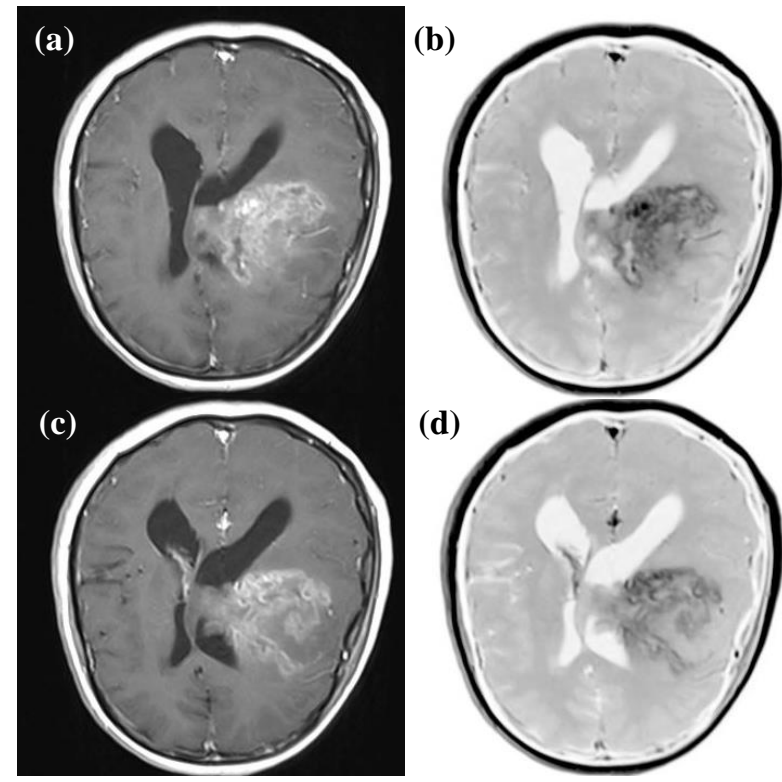

Fig 4: The original MRI is shown in (a) and in (c) and the signal resilient to interpolation is shown in (c) and in (d). The level of details of the tumor region is equally visible in the signal resilient to interpolation images in (b) and in (d) when compared to the MRI in (a) and in (c). The dark region is presumably blood and/or water. The matrix size of the images is $512 \times 512$ with pixel size of $0.49 \mathrm{~mm} \times$ $0.49 \mathrm{~mm}$. The images in (a) and (c) were fitted with the bivariate cubic Lagrange polynomial and they were re-sampled of the misplacement (x, $\mathrm{y})=(0.01 \mathrm{~mm}, 0.01 \mathrm{~mm})$

An interesting observation visible in Fig. 3 is that the Cerebrospinal Fluid (CSF) is imaged with the same color (in dark color) of the fluids of the tumor (see (a) and (c)), and as far as regards this, the opposite is visible (in bright color) in the images of the signal resilient to interpolation in Fig. 3b and in Fig. 3d.

The dark nuances seen in Fig. 4 demonstrate, as far as regards the signal resilient to interpolation images seen in (b) and in (d), that the level of details of the tumor is quite similar to the MRI image seen in (a) and in (c) respectively. The images in Fig. 4 have been adjusted to the level of brightness-contrast enhancement so to make an objective comparison. The tendency seen in Fig. 2, Fig. 3 and Fig. 4 is though, that one of complementariness of the signal resilient to interpolation to the MRI, whereas in Fig. 1 the tendency of the signal resilient to interpolation is that one of offering additional information about the tumor to the MRI.

\section{B. The Intensity Curvature Functional}

Fig. 5 shows the combined use of the signal resilient to interpolation and the Intensity-Curvature Functional. The signal resilient to interpolation was calculated when fitting the parametric bivariate quadratic B-Spline model function [21-23] (see (c) and (d)) to the original MRI shown in (a) and in (b), whereas the Intensity-Curvature Functional (see (e) and (f)) was calculated when fitting the bivariate linear model function. The misplacement used to re-sample the images so to obtain Fig. 5c and Fig. $5 \mathrm{~d}$ is $(\mathrm{x}, \mathrm{y})=(0.9 \mathrm{~mm}, 0.9 \mathrm{~mm})$, with the value of the parametric constant set to 2.54 , and the misplacement used to re-sample the images so to obtain the images seen in Fig. $5 e$ and in Fig. $5 \mathrm{f}$ is $(\mathrm{x}, \mathrm{y})=(0.01 \mathrm{~mm}, 0.01 \mathrm{~mm})$.
Fig. 6 shows the Intensity-Curvature Functional calculated on the basis of the original MRI seen in Fig. 1a and Fig. 1c. As anticipated earlier the main characteristic of the Intensity-Curvature Functional is that one of displaying the third dimension perpendicular to the image plane.

The third dimension reveals information about the anatomical structures of the human brain and as such also the tumor structures are highlighted. In this section of the paper, the Intensity-Curvature Functional was calculated when fitting to the MRI the bivariate linear model function and the intra-pixel re-sampling coordinate is: ( $\mathrm{x}$, y) $=(0.5 \mathrm{~mm}, 0.5 \mathrm{~mm})$. An important characteristic shown in Fig. 7 is the highlight of the fluids of the tumor.

When looking at Fig. 7 in comparison to Fig. 2a and Fig. 2b, it is possible to recognize that what was seen in the aforementioned pictures in bright and dark is here reproduced with an elevation from the imaging plane and such elevation adds information to the original MRI as far as regards the anatomy of the tumor structures. In general, it is visible from the pictures reported in this section that the most important capability of the Intensity-Curvature Functional is that one of presenting information which is not observable in the original MRI and such information pertains to the third dimension given by the IntensityCurvature Functional to the anatomical structures of the brain and more specifically to the anatomy and the details of the tumor.
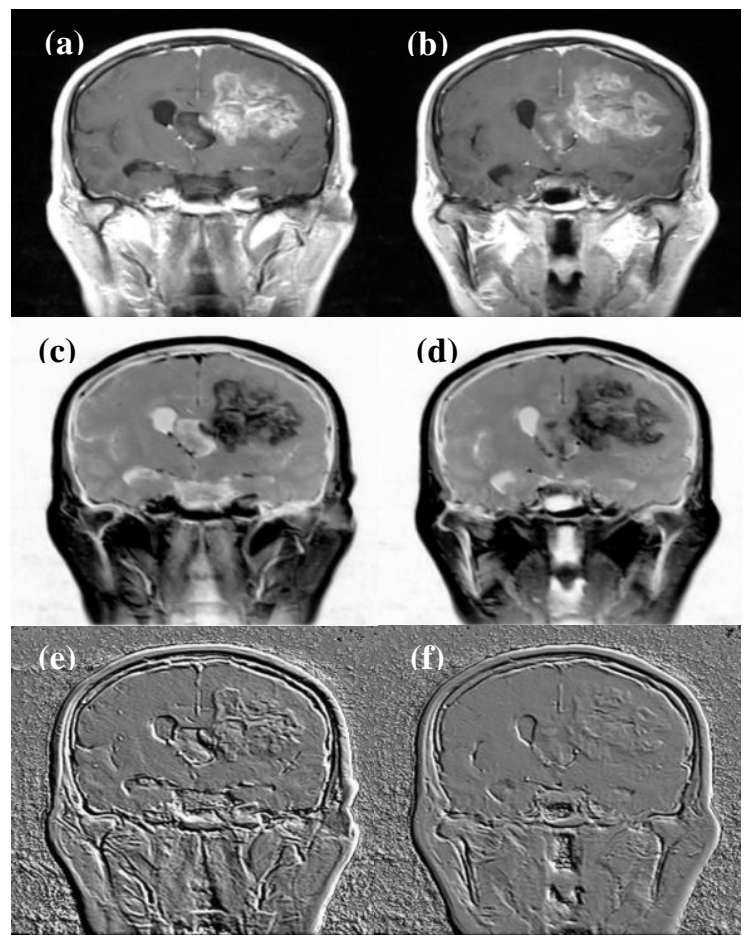

Fig 5: The original MRI is shown in (a) and in (b). The signal resilient to interpolation is located in the images shown in (c) and in (d). The Intensity-Curvature Functional is shown in (e) and in (f). The matrix size of the images is $512 \times 512$ with pixel size of $0.45 \mathrm{~mm} \times$ $0.45 \mathrm{~mm}$. While the signal resilient to interpolation clearly shows complementary information to the original MRI, the Intensity-Curvature Functional adds information to the original MRI when showing the depths of the anatomical structures of the tumor. The depths are more visible in (e) than in (f). The images were cropped so to highlight the regions of interest. 


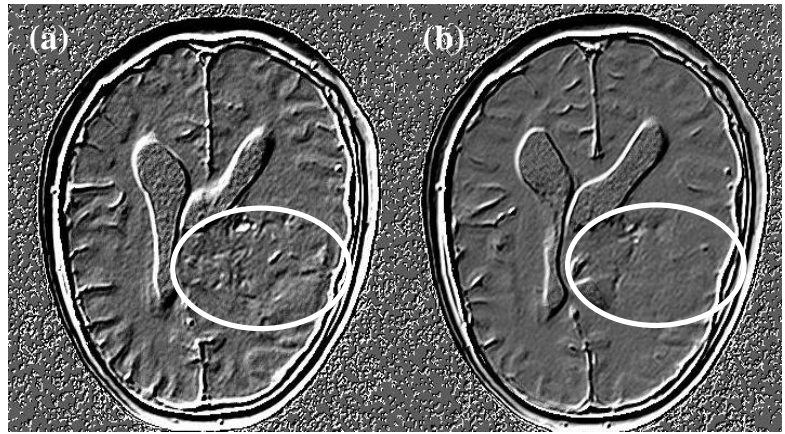

Fig 6: The Intensity-Curvature Functional of the MRI seen in Fig. 1a is shown in (a), whereas the Intensity-Curvature Functional of the MRI seen in Fig. 1c is shown in (b). The elevation of the cortex with respect to the cerebrospinal fluid (CSF) is visible from the pictures and also the contour line of the ventricles appears as such to give the perception of the third dimension perpendicular to the image plane. The tumor structures are highlighted in the regions inside the white ellipses (see Fig. 1a and Fig. 1c for reference).

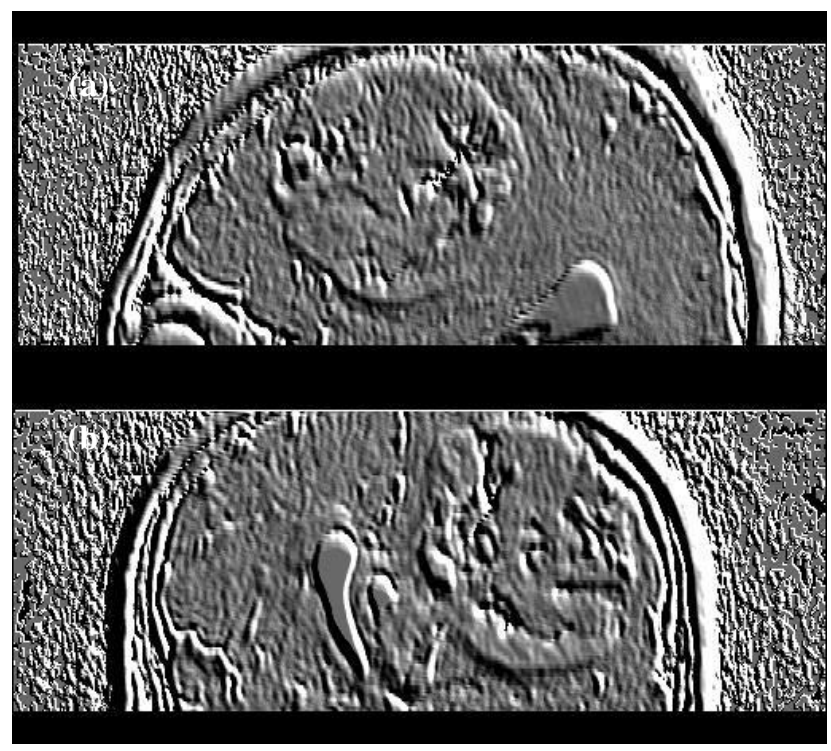

Fig 7: The Intensity-Curvature Functional of the MRI shown in Fig. $2 \mathrm{a}$ is on display in (a), whereas the Intensity-Curvature Functional of the MRI shown in Fig. 2c is shown in (b). The characteristics of the images in (a) and in (b) is that of presenting the third dimension of the tumor structures, along with the CSF (see (b)), which appears elevated along the axis perpendicular to the imaging plane.

Clearly, Fig. 7 shows that the Intensity-Curvature Functional is capable to identify the tumor contour line. In Fig. 8 the accent is on the ventricles, the CSF and the anatomical structures of the tumor. The pressure exercised by the tumor on the ventricles and the third dimension of the tumor structures are visible. Specifically, what was seen in white (most presumably fluids) in Fig. $3 \mathrm{a}$ and in Fig. 3c is here seen in Fig. 8a and in Fig. 8b with an elevation from the image plane. It is worth mentioning that the combined information provided through the signal resilient to interpolation images seen in Fig. $3 \mathrm{~b}$ and in Fig. 3d, which is complementary to the original MRI, is now corroborated with the additional information provided through the Intensity-Curvature Functional shown in Fig. 8a (calculated from Fig. 3a) and the Intensity-Curvature Functional shown in Fig. 8b (calculated from Fig. 3c).

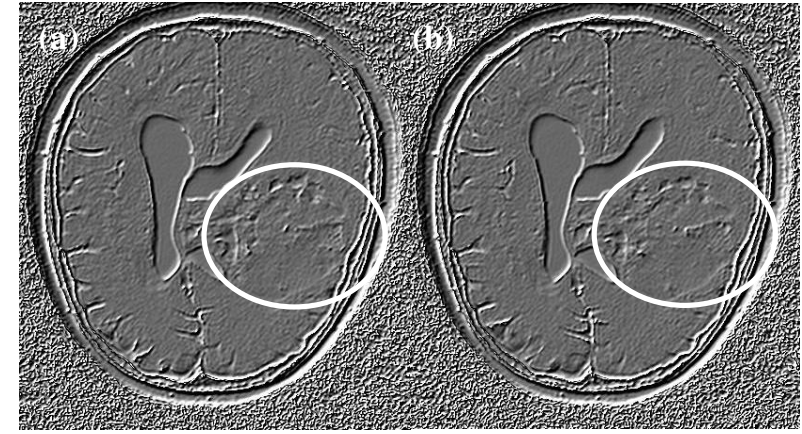

Fig 8: The Intensity-Curvature Functional of Fig. 3a is shown in (a) and the Intensity-Curvature Functional of Fig. 3c is shown in (b). These pictures present another evidence of the capability of the IntensityCurvature Functional to build the third dimension perpendicular to the image plane. In the specifics, the ventricles and the CSF are shown with an elevation when compared to the cortex and more specifically when compared with the anatomy of the tumor (see regions inside the white ellipses).

The additional information provided through the Intensity-Curvature Functional images shown in Fig. 9 is relevant to the white nuances seen in Fig. 4a and Fig. 4c. The Intensity-Curvature Functional in the case presented in Fig. 9 is able to build a third dimension on the basis of what in Fig. 4 are shown as fluids of the tumor. This is not the first instance as it was seen in the previous figures seen in this section, which show that the fluids can have a third dimension extracted by the Intensity-Curvature Functional.

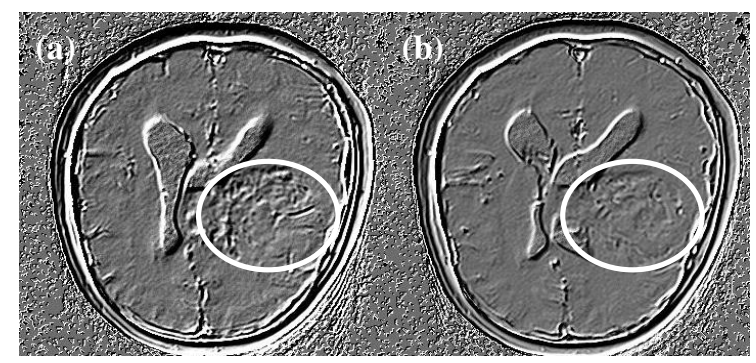

Fig 9: The Intensity-Curvature Functional of the MRI shown in Fig. $4 \mathrm{a}$ is shown in (a), and the Intensity-Curvature Functional of the MRI shown in Fig. 4c is shown in (b). A remarkable feature of the IntensityCurvature Functional images is the making of the third dimension of the white nuances seen in Fig. 4 (see regions of the tumor inside the white ellipses). Also, the contour line of the ventricles is elevated from the inner CSF and the cortical region appears at the same elevation of the ventricles (see the contour white line).

\section{DISCUSSION}

The major findings of the research herein presented are congruent with the following concept. An image processing algorithm which is intended to perform feature extraction from the original image cannot extract any realities from the original image which do not exists in the image. Think of the previously stated concept likewise filter theory. A filter applied to a signal can extract frequencies located in the signal but cannot make frequencies out of the signal that are not located in the signal. The congruency of the aforementioned logic is in 
harmony with the results seen from the calculation of the signal resilient to interpolation from the original MRI. We have seen through the results presented in this paper that the tendency of the signal resilient to interpolation is to reproduce the MRI with similar, if not equal, level of details. However the reproduction of the details of the signal resilient to interpolation happens in a domain which is not the same as the one of the MRI, and thus it was seen that the signal resilient to interpolation adds complementary but not additional information to the original MRI. Nevertheless, the true novelty in this paper is the capability of the Intensity-Curvature Functional [24, 25 ] to perform feature extraction from the MRI and to make of the MRI a new domain where details are shown, which are not readily observable in the original domain. The previously stated novelty breaks the grounds of the congruency recalled at the beginning of this discussion and proposes the Intensity-Curvature Functional as the mathematical engineering tool capable to extract realities in the images which would not be observable otherwise. The question for the future is still along the line of thought of the congruency of this research with the theoretical statement that forbids the creation of realities from non-existing ones. In other words, the question for the future of the herein presented study is in the making of the additional evidence that the reality created by the Intensity-Curvature Functional is indeed present already in the original MRI and needs to be extracted. The third dimension seen in the Intensity-Curvature Functional images, however, at the present stage of the research, breaks the grounds of congruency and brings the results to a higher level of testable validity. Should the third dimension seen in the images through the IntensityCurvature Functional belong to a reality not located in the MRI, then the Intensity-Curvature Functional is not a feature extractor but an instrument placed on a different level of congruency as far as regards the one to which the signal resilient to interpolation belongs to.

One aspect which is relevant to the methodological approach to the diagnosis made on the basis of the aid of the signal resilient to interpolation and the IntensityCurvature Functional to the MRI is the preparedness of the physician reading the images. In the cases presented in this paper, the constant parameter of the polynomial model functions was set to the value of 2.54 for the bivariate quadratic B-Spline polynomial, and to the value of -2.54 for the bivariate cubic Lagrange polynomial. The signal resilient to interpolation images tend to appear as the specular negative of the original MRI. This fact needs to have the physician ready to interpret the signal resilient to interpolation images but the advantage is that the evaluation of the signal resilient to interpolation images happens in parallel with the MRI. Although not reported here, it is possible to obtain signal resilient to interpolation images with the gray scale level consistent with that one of the MRI. For instance signal resilient to interpolation images which are very much the same as the MRI images.

A few remarks should be made concerning the following observation. Fig. 1c, Fig. 3c and Fig. 4c have their Intensity-Curvature Functional calculated and displayed in Fig. 6b, Fig. 8b and Fig. 9b respectively. As it can be noted the aforementioned Intensity-Curvature Functional figures show a region of notable level of flatness. Such region is corresponding in the original MRI to a region where the tumor structures are also flat. Conversely, it is possible to observe a notable tendency of the Intensity-Curvature Functional images to display the third dimension in correspondence to the MRI images having complex structures made of, or surrounded by, tumor fluids such as water and blood.

An interesting behavior is discernible in the signal resilient to interpolation of the MRI shown in Fig. 2a, Fig. 2c, Fig. 5a, Fig. 5b, and the Intensity-Curvature Functional of Fig. 7a and Fig. 7b. While in Fig. 2 the signal resilient to interpolation responds with the specular negative imaging shown in Fig. 2b and in Fig. 2d, in Fig. 5 and in Fig. 7 the Intensity-Curvature Functional responds with a clear and neat third dimension. In Fig. 5 and in Fig. 7 the tumor shows anatomical structures as well as nuances attributable to fluids. In both of the cases seen in Fig. 5 and in Fig. 7 the Intensity-Curvature Functional responds similarly, thus extracting the third dimension.

\section{CONCLUSION}

The most important benefit of the signal resilient to interpolation and the Intensity-Curvature Functional is to provide the diagnostic settings with two imaging instruments which expand the capability of MRI. This benefit was explored in this research through the study of an MRI tumor case.

More benefits of the combined use of the signal resilient to interpolation and the Intensity-Curvature Functional yields the obtainment of images which favors, on the basis of the original MRI, both: (i) the reproduction under a different perspective, and (ii) the making of a different reality. The results presented in this piece of research, which studies a tumor case, show that the combined use of the signal resilient to interpolation and the Intensity-Curvature Functional places the emphasis on the anatomy of the tumor, the fluids (most presumably water and blood) of the tumor and the nuances of the pathology. Overall, the role of the signal resilient to interpolation, which was seen through the results presented herein, show complementary information to the original MRI, whereas the IntensityCurvature Functional show additional information to the original MRI.

On a theoretical basis, one benefit of the signal resilient to interpolation and the Intensity-Curvature Functional is the fact that both of them can be calculated on any model polynomial function which has of the property of second order differentiability.

As far as the limitations are concerned, the one which is most relevant is the complexity of the mathematical procedures which are necessary to formulate both of the signal resilient to interpolation and the IntensityCurvature Functional. Nevertheless, the software 
implementation of the math formulations is also a limitation, because of the length of the math formulae.

\section{ACKNOWLEDGMENT}

The software is available from the corresponding author at no charge whatsoever. This work presents a case study on a patient suffering from brain tumor. The MRI scans were collected after proper administration of the informed consent to the patient and in agreement with ethical committees of the Skopje City General Hospital. The authors are grateful to Ms. Monica Chiang because of the coordination of the paper submission, to the anonymous reviewers because of their useful suggestions aimed to improve the paper, and to the MECS Publisher editorial team because of the excellent typesetting of the paper.

\section{REFERENCES}

[1] C. Ciulla, "Signal resilient to interpolation: An exploration on the approximation properties of the mathematical functions", CreateSpace Publisher, U.S.A., 2012.

[2] C. Ciulla, "Improved signal and image interpolation in biomedical applications: The case of magnetic resonance imaging (MRI)", Medical Information Science Reference IGI Global Publisher, Hershey, PA, U.S.A., 2009.

[3] S. Ouadfel, and S. Meshoul, "A Fully adaptive and hybrid method for image segmentation using multilevel thresholding", International Journal of Image, Graphics and Signal Processing, vol. 5, no. 1, pp. 46-57, 2013.

[4] N. Sumitra, and R. K. Saxena, "Brain tumor classification using back propagation neural network", International Journal of Image, Graphics and Signal Processing, vol. 5, no. 2, pp. 45-50, 2013.

[5] M. A. Amirkhizi, and S. Haghipour, "Left ventricle segmentation in magnetic resonance images with modified active contour method", International Journal of Image, Graphics and Signal Processing, vol. 5, no. 10, pp. 19-25, 2013.

[6] V. Pathak, P. Dhyani, and P. Mahanti, “Autonomous image segmentation using density-adaptive dendritic cell algorithm", International Journal of Image, Graphics and Signal Processing, vol. 5, no. 10, pp. 26-35, 2013.

[7] A. B. Al-Othman, Z. Al-Ameen, and G. B. Sulong, "Improving the MRI tumor segmentation process using appropriate image processing techniques", International Journal of Image, Graphics and Signal Processing, vol. 6, no. 2, pp. 23-29, 2014.

[8] K.M. Iftekharuddin, J. Zheng, M.A. Islam, and R.J. Ogg, "Fractal-based brain tumor detection in multimodal MRI", Applied Mathematics and Computation, vol. 207, no. 1, 23-41, 2009

[9] A. Islam, S.M.S. Reza, and K.M. Iftekharuddin, "Multifractal texture estimation for detection and segmentation of brain tumors", IEEE Transactions on Biomedical Engineering, vol. 60, no. 11, 3204 - 3215, 2013.

[10] Y. Zhu, and H. Yan, "Computerized tumor boundary detection using a Hopfield neural network", IEEE Transactions on Medical Imaging, vol. 16, no. 1, 55-67, 1997.
[11] M. Prastawa, E. Bullitt, N. Moon, K. Van Leemput, and G. Gerig, "Automatic brain tumor segmentation by subject specific modification of atlas priors", Academic Radiology, vol. 10, no. 12, 1341-1348, 2003.

[12] M.C. Clark, L.O. Hall, D.B. Goldgof, R. Velthuizen, F.R. Murtagh, and M.S. Silbiger, "Automatic tumor segmentation using knowledge-based techniques", IEEE Transactions on Medical Imaging. vol. 17, no. 2, pp. 187201, 1998.

[13] R. B. Dubey, M. Hanmandlu, S. K. Gupta, and S.K. Gupta, "Semi-automatic segmentation of MRI Brain tumor", ICGST-GVIP Journal, vol. 9, no. 4, pp. 33-40, 2009.

[14] D. T. Gering, W. E. L. Grimson, and R. Kikinis, Recognizing deviations from normalcy for brain tumor segmentation, Springer Berlin Heidelberg, pp. 388-395, 2002.

[15] A. Hamamci, G. Unal, N. Kucuk, and K. Engin, "Cellular automata segmentation of brain tumors on post contrast MR images", In: Medical Image Computing and Computer-Assisted Intervention - MICCAI, Springer Berlin Heidelberg, pp. 137-146, 2010.

[16] A. Hamamci, N. Kucuk, K. Karaman, K. Engin, G. Unal, "Tumor-cut: segmentation of brain tumors on contrast enhanced MR images for radiosurgery applications", IEEE Transactions on Medical Imaging, vol. 31, no. 3, pp. 790804, 2012.

[17] M. M. Letteboer, O. F. Olsen, E. B. Dam, P. W. Willems, M. A. Viergever, and W. J. Niessen, "Segmentation of tumors in magnetic resonance brain images using an interactive multiscale watershed algorithm", Academic Radiology, vol. 11, no. 10, pp. 1125-1138, 2004.

[18] S. Ruan, B. Moretti, J. Fadili, and D. Bloyet, "Fuzzy Markovian segmentation in application of magnetic resonance images", Computer Vision and Image Understanding, vol. 85, no. 1, pp. 54-69, 2002.

[19] S. Shen, W. Sandham, M. Granat, and A. Sterr, "MRI fuzzy segmentation of brain tissue using neighborhood attraction with neural-network optimization", IEEE Transactions on Information Technology in Biomedicine, vol. 9, no. 3, pp. 459-467, 2005.

[20] Y. Zhang, M. Brady, and S. Smith, "Segmentation of brain MR images through a hidden Markov random field model and the expectation-maximization algorithm", IEEE Transactions on Medical Imaging, vol. 20, no. 1, pp. 45-57, 2001.

[21] C. De Boor, "A practical guide to splines" Applied mathematical sciences, Springer-Verlag, 1978.

[22] M. Unser, A. Aldroubi, and M. Eden, "B-spline signal processing: Part I - theory", IEEE Transactions on Signal Processing, vol. 41, no. 2, pp. 821-833, 1993a.

[23] M., Unser, A. Aldroubi, and M. Eden, "B-spline signal processing: Part II - efficient design and applications", IEEE Transactions on Signal Processing, vol. 41, no. 2, pp. 834-848, 1993 b.

[24] C. Ciulla, "The intensity-curvature functional of the trivariate cubic Lagrange interpolation formula", International Journal of Image, Graphics and Signal Processing, vol. 5, no. 10, pp. 36-44, 2013.

[25] C. Ciulla, "On the signal-image intensity-curvature Content", International Journal of Image, Graphics and Signal Processing, vol. 5, no. 5, pp. 15-21, 2013. 


\section{AUTHORS' PROFILES}

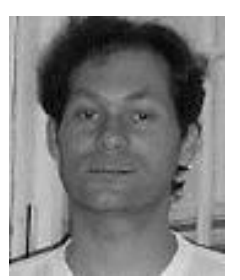

Carlo Ciulla had been undergraduate and graduate student at the University of Palermo, Italy, RUTGERS University, U.S.A. and the New Jersey Institute of Technology, U.S.A. from 1987 to 2002 . He has earned the following graduate degrees: Laurea in Management Engineering (1994, Italy); an M.S. in Information Systems and a Ph.D. in Computer and Information Science (2000, 2002, U.S.A.). Following the completion of the Doctoral degree, Carlo's former academic appointments were: Research Associate at Yale University (2002-2003); Postdoctoral Scholar at the University of Iowa (2004-2005); Postdoctoral Scholar at Wayne State University (2005-2007); Assistant Professor of Computer Science at Lane College (2007-2009). During the years 2009-2012 Carlo was a self-employed scholar that devoted his time to his research interest related to the development of innovative methods of signal interpolation and also to the development of educational software for students. In September 2012 Carlo joined as Assistant Professor of Computer Science, the University for Information Science and Technology in Ohrid, Macedonia.

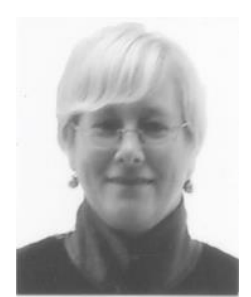

Dijana Capeska Bogatinoska is a teaching and research assistant of Computer Science and Engineering at the University for Information Science and Technology "St. Paul the Apostle" Ohrid, Macedonia. She received her M.S. in Computer Science from the University "St. Clement of Ohrid" - Technical Faculty Bitola, and the B. Eng. in Electrical Engineering from the Military Technical Faculty - Belgrade, in the Department of Electronics. Dijana has contributed a considerable number of manuscripts to conferences and congresses in the field of information science and technologies. Currently she is Ph.D. candidate at the University "St. Clement of Ohrid" - Technical Faculty Bitola, in the Computer Science and Engineering Department. She has previous working experience at Eurotec in Prilep, Macedonia, as engineer for preparing printed circuit boards for production, in Euroinvest 11 Oktomvri in Prilep, Macedonia, first as a programmer at the computer science department, later as the head of the computer science department and finally as the manager of the financial and administrative department.

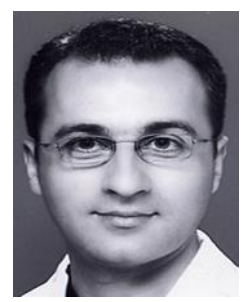

Filip A. Risteski was awarded the degree of Physician at the Faculty of Medicine, St. Cyril and Methodious University in Skopje, Macedonia in 2005. Following the completion of the degree of Physician, Dr. Risteski earned the position of Medical Specialist in Radiology from the Radiology Institute at the Faculty of Medicine, St. Cyril and Methodious University in Skopje, Macedonia. Dr. Risteski is now with the Skopje City General Hospital, Skopje, Macedonia since 2011. During the years 2002-2009 Dr. Risteski has been an active contributor of scientific articles in the field of applied radiology, and he is currently a member of the Macedonian Medical Association, the Macedonian Radiologists Association, the European Society of Radiology, the European Society of Skeletal Radiology and the Radiological Society of North America.

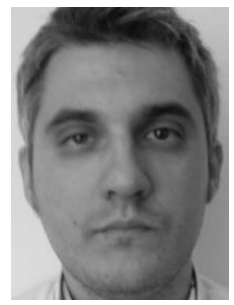

Dimitar Veljanovski was awarded the degree in General Medicine from the Medical Academy, Sofia, Faculty of Medicine in 2006. During the years 2007 through 2011 Dr. Dimitar Veljanovski has been working on his specialization in diagnostic radiology at the Clinic of Radiology in the Clinical Center in Skopje. During the years 2003 through 2013, Dr. Dimitar Veljanovski has contributed a considerable number of manuscripts to conferences and symposiums and has also attended numerous training and seminars in the field of diagnostic radiology. Since August 2011, Dr. Dimitar Veljanovski is with the Skopje City General Hospital in the Department of Magnetic Resonance Imaging and he is an active member of the Macedonian Radiology Association and the European Radiology Society.

Manuscript received January $21^{\text {st }}, 2014$; revised March $1^{\text {st }}$, 2014; accepted Month Date, Year.

How to cite this paper: Carlo Ciulla, Dijana Capeska Bogatinoska, Filip A. Risteski, Dimitar Veljanovski,"Applied Computational Engineering in Magnetic Resonance Imaging: A Tumor Case Study", IJIGSP, vol.6, no.7, pp.1-9, 2014.DOI: $10.5815 /$ ijigsp.2014.07.01 